\title{
IN-SITU COMPRESSION TEST OF ARTIFICIAL BONE FOAMS IN CONTROLLED ENVIRONMENT USING X-RAY MICRO-COMPUTED TOMOGRAPHY
}

\author{
Jonathan Glinz ${ }^{a, *}$, DANiel KYTÝŘ ${ }^{b}$, TOMÁŠ Fíla ${ }^{b}$, JAN ŠleichrT ${ }^{b}$, \\ Andreas SchrempF ${ }^{c}$, DAvid FÜrst $^{c}$, Johann KAStner $^{a}$, SAscha SencK $^{a}$ \\ ${ }^{a}$ University of Applied Sciences Upper Austria, Stelzhamerstraße 23, 4600 Wels, Austria \\ ${ }^{b}$ Czech Academy of Sciences, Institute of Theoretical and Applied Mechanics, Prosecká 809/76, 19000 Prague 9, \\ Czech Republic \\ ${ }^{c}$ University of Applied Sciences Upper Austria, Garnsionstrasse 21, 4020 Linz, Austria \\ * corresponding author: jonathan.glinz@fh-wels.at
}

\begin{abstract}
In this study, we investigated specimens of artificial bone foams, developed by the research group for surgical simulators at the UAS Linz, which are used to mimic the haptic feedback of physiologic and pathologic bone for more realistic surgery training. Specimens with two kinds of mineral filler material as well as different amounts of foaming agent were tested in an environmental in-situ loading stage developed by the ITAM CAS and scanned via X-ray micro-computed tomography. In this in-situ stage, specimens can be immersed in liquid and tested under temperature-controlled conditions. Consequently, a total amount of 12 specimens was subjected to compression loading; half of them immersed in water at $36.5^{\circ} \mathrm{C}$ and half in dry condition. Results showed that there is no significant influence of liquid immersion to the compression outcome. However, foams with less amount of foaming agent appeared to have smaller pores resulting in higher compression strength.
\end{abstract}

KEYwORDs: X-ray micro-computed tomography, artificial bone foams, in-Situ testing.

\section{INTRODUCTION}

The hybrid patient simulator devices developed by the research group for surgical simulators (ReSSL) at the UAS Linz use artificial bone foams to recreate the haptic feedback of human bone. These devices should thereby provide the possibility to train surgical procedures such as the application of pedicle screws and bone cement in a safe environment [1]. Since excessive hours of training are essential to build up the experience necessary for surgeons, with this approach of a simulated patient, the use of actual specimens from human or animals can be reduced and cost as well as ethical questions satisfied [2. However, because of the complex structure of bone and bone replacement materials, pore morphology and mechanical properties have to be investigated and visualized in a three-dimensional way in order to guarantee for characteristics comparable to actual human bone [3, 4, E.g. simulation experiments by means of finite element methods can be performed to evaluate material parameters in a non-destructive (NDT) way. However, the reliability of such experiments highly depends on the accuracy of the acquired volume data. X-ray micro-computed tomography (XCT) has established as a standard NDT method, which offers sufficient accuracy for the investigation of such materials [5]. For an in-depth characterization of histomorphometric features, digital volume data acquired by XCT can be processed and visualized three dimensionally to determine bone volume fraction, cortical thickness and porosity. Similar specimen of artificial bone foam developed by have already been characterized precisely in [6, 7]. However, these specimens were tested only in a dry state. Since physiological bone typically is in a wet state, the main purpose of this study was the investigation of the influence of environmental conditions on artificial bone foams of varying composition. To this end, non-destructive XCT in combination with destructive compression tests were carried out.

\section{Materials And Methods}

\subsection{SAMPLE PREPARATION}

In the course of this study, artificial bone foam specimens made from polyurethane resin with two different kinds of mineral filler material $\left(\mathrm{CaCO}_{3}\right.$, $\left.3 \mathrm{Ca}_{3}\left(\mathrm{PO}_{4}\right) \cdot \mathrm{CaO}\right)$ were produced. This mineral fillers were added in order to increase the density of the foams to match that of physiological bone more closely. To start the foaming process, water from 0.5 to $1.0 \%$ by weight was added as blowing agent. The ingredients were mixed and filled into casts where the foam structure developed by chemical reaction. The test specimens were cut out of these casted foams using hollow-core drills. The final dimensions of the specimens are $\varnothing 12 \times 20 \mathrm{~mm}$ as visible in Fig. 1 .

\subsection{Mechanical Testing}

The specimens were tested in an in-situ loading stage, shown in Fig. 2, which has been developed by the ITAM CAS. The device consists of aluminum main 


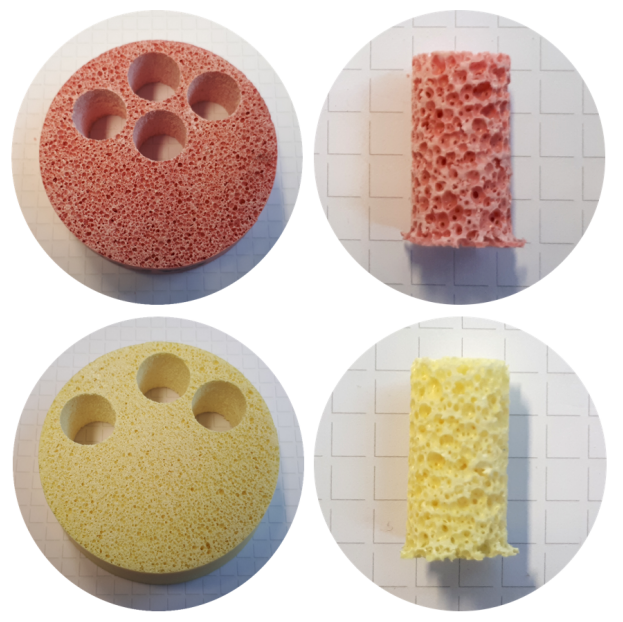

Figure 1. Examples of CaP (red) and $\mathrm{CaC}$ (yellow) foams as casted (left) and the final specimens after hollow-core drilling (right).

frame, hollow shaft stepper motor, harmonic gearbox (with standard accuracy about 1.5 arcmin) and carbon tube equipped with a upper support. In this stage, specimens can be immersed in liquid and tested under temperature-controlled conditions also. Consequently, a total amount of 12 specimens were subjected to compression loading; half of them immersed in water at $36.5^{\circ} \mathrm{C}$ and half in dry condition. For all displacementdriven tests, a uniform value of the loading rate was set to $1 \mathrm{~mm} / \mathrm{min}$ and the experiments were performed up to $\approx 70 \%$ of deformation. The position of the moving support was declared by a magnetic linear encoder with the resolution of $20 \mu \mathrm{m}$. Values of the actual force, performed by a loadcell with nominal capacity of $500 \mathrm{~N}$ (HBM U9b, Germany) was recorded by a multifunction data acquisition device. The motion of the system and all other peripherals are controlled using a custom developed control software based on the open-source project LinuxCNC running on the real-time kernel [8]. The control hardware consists of high-performance motion control components (MESA Electronics, U.S.A.), sensor readout electronics (LabJack T7, U.S.A.) and custom electronics. Furthermore, a time-lapse in-situ investigation with $\mathrm{XCT}$ scans in-between the load steps was performed for one specimen immersed in water to investigate behavior during load.

\subsection{Micro-COMPUted TOMOGRAPHY}

The XCT scans were performed on a RayScan 250E system equipped with a $225 \mathrm{kV}$ and $450 \mathrm{kV} \mathrm{X}$-ray source and a $2048 \times 2048$ pixels flat panel detector as visible in Fig. 3. For this study only the $225 \mathrm{kV}$ source was utilized.

To investigate the morphological changes caused by the compression of a specimen, a total amount of $6 \mathrm{XCT}$ scans was performed. The scanning parameters was set according to maximize signal to noise ration and to obtain reliable statistics for proper volumetric dataset reconstruction [9]. The first scan

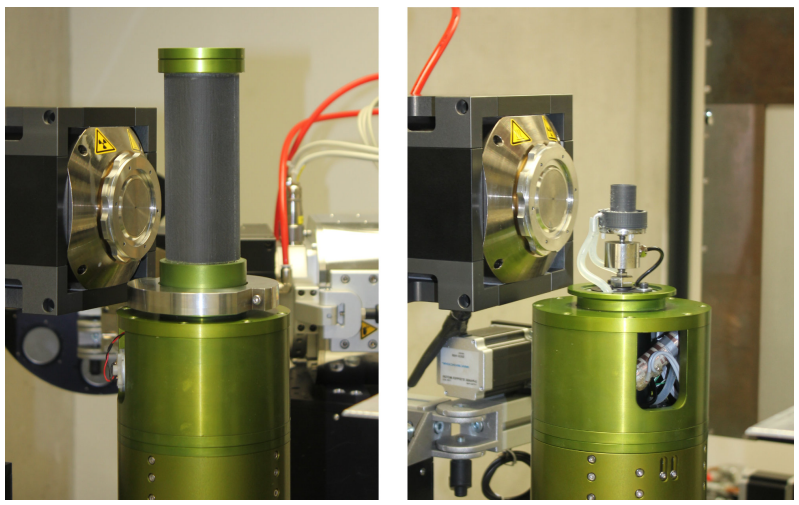

Figure 2. Environmental in-situ stage in closed carbon tube (left) and open state (right).

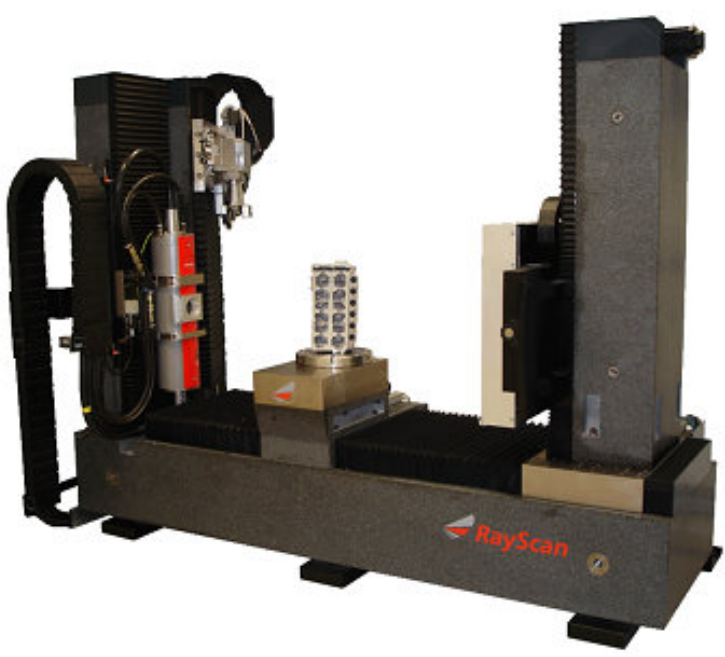

Figure 3. Image of the RayScan 250E system with dual sources on the left and flat panel detector on the right. An exemplary specimen is positioned on the rotary stage in-between.

was recorded during the initial, unloaded state of the specimen, followed by a scan after every load step applied. To prevent motion artefacts, 30 minutes of relaxation time was scheduled after every load step before starting the next scan to allow for sufficient stabilization of the sample. With the diameter given by the environmental stage, we were able to achieve a minimum voxel size of $10 \times 10 \times 10 \mu \mathrm{m}^{3}$. A tube voltage of $90 \mathrm{kV}$ at a current of $115 \mathrm{kV}$ was chosen to penetrate the samples inside the carbon tube of the in-situ stage. The XCT scans were performed at a total amount of 1260 projections with an exposure time of $666 \mathrm{~ms}$ each. No additional pre-filter plates or averaging was applied. This sums up to a total scan time of 54 minutes for each scan.

\subsection{IMAGE PROCESSING}

For the visualization of water immersion, a $5 \times 5 \times 5$ median filter was applied to get rid of the most severe image noise. However, a quantification of water filled pore volume was not possible because of the low contrast between water and the artificial bone foam. 


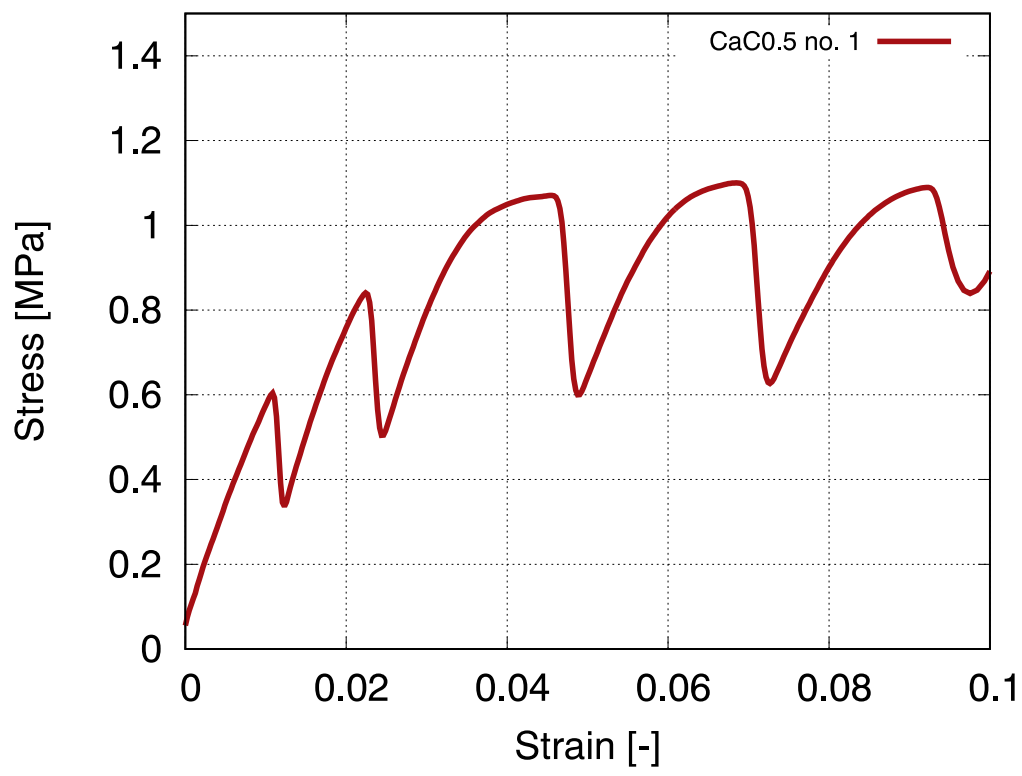

FigURE 4. Resulting stress-strain diagram of the time-lapse in-situ investigation performed on a $\mathrm{CaCO}_{3}$ filler specimen with $0.5 \%$ foaming agent. The first two loading steps were performed with $0.6 \%$ strain increment followed by $1.2 \%$ steps.

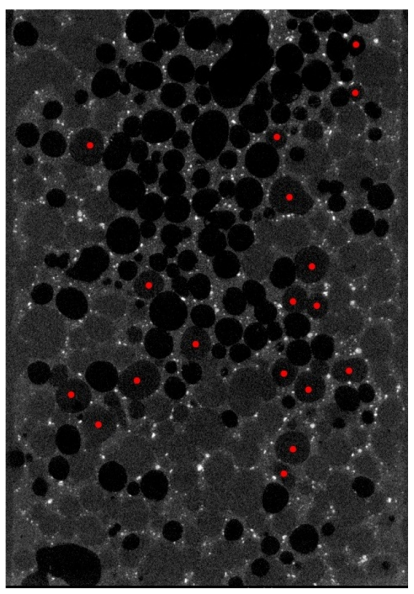

$0-1$

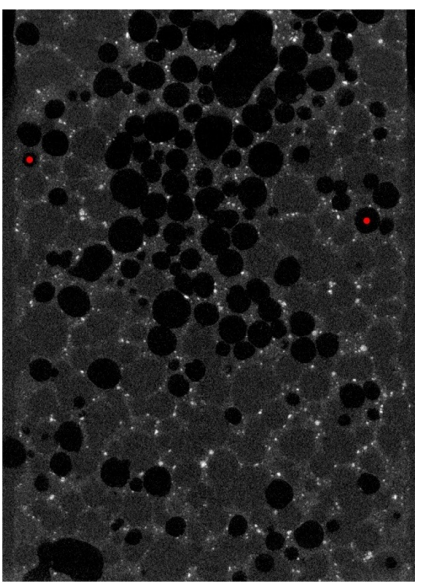

$1-4$

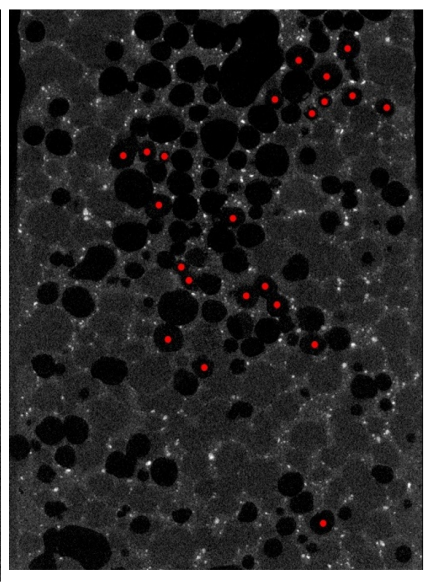

4-5

Figure 5. Cross section images of the time-lapse in-situ investigation. Pores that filled with water between the load steps are indicated with red dots.

Therefore, the investigation of permeability was performed by measurement of remaining air inside the specimen. Pores filled with air were segmented by an ISO50 threshold between the air and bone foam material peak in the grey value histogram [10. Eventually detected bubbles of air outside of the bone foam material were removed manually from the data.

\section{Results and Discussion}

The force resistance resulting from the time-lapse insitu experiments is visible in Fig. 4. It can be seen that the maximum force response increases during the early load steps while steps from position 2 to 5 result in an approximately constant maximum forces.

In accordance with these results, Fig. 5 shows cross sectional images of the specimen at position 0 (initial state), 1 and 4. Despite the open porous morphology of the foam, water immersed only partially into the foam, leaving pores closer to the center unfilled. Pores indicated with red dots are empty in the shown position but fill with water in the course of the experiment. It can be seen, that during the first and last load steps a higher amount of water ingresses into the pores than from position 1 to 4 . This indicates that more time for the intrusion of water into the pores is necessary at the beginning of the investigations. Furthermore, the higher intrusion of water during the final load step might indicate that at this state deformation has already exceeded the elastic range and cell walls are being destroyed. In numbers, the volume of air filled pores was reduced by $15.82 \%$ after the first load step and only by additional $0.73 \%$ until position 4 . During the final load step, air volume again decreased by $13.56 \%$. 
The resulting forces during the continuous compression experiments performed on 12 samples are shown in Fig. 6. Each material composition (filler material and amount of foaming agent) was tested in dry condition and immersed in water. Since both, dry and wet conditions, lead to comparable force response, no significant influence of liquid immersion to the compression outcome could be observed. However, foams with less amount of foaming agent show higher forces. This can be explained by a reduced average pore size resulting in higher compression strength. Although one might argue that the CaP filled sample with $0.75 \%$ foaming agent shows slightly higher forces in comparison to the $\mathrm{CaC}$ filled, a general trend of $\mathrm{CaP}$ filler material leading to higher compression strength cannot be confirmed

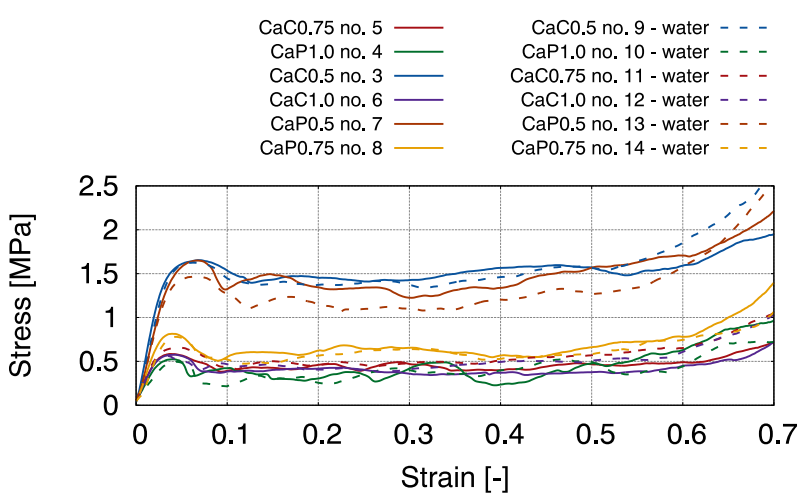

FIGURE 6. Stress-strain diagram of all tested specimens. Dry specimens are shown in solid and specimens immersed in water as dashed lines.

\section{Conclusions}

Concluding, the usage of the artificial bone foams investigated is despite their physiologically wet condition also valid in a dry state since environmental differences are nonessential for their mechanical properties. However, to confirm the influence of different filler materials on the mechanical properties of the foams, additional measurements with a larger sample size for statistical evaluation are necessary. Furthermore, it must be considered that the influence of water immersion to the compression force results might be altered since not all pores were filled with water during the tests. For future studies, longer waiting times for the immersion of water or a pressurized intrusion should be considered.

\section{ACKNOWLEDGEMENTS}

The research has been supported by the European Regional Development Fund in frame of the projects Competence Center for High-Resolution 3D X-ray Imaging (ATCZ38) in the Interreg V-A Austria - Czech Republic programme and by Operational Programme Research, Development and Education in project INAFYM (CZ.02.1.01/0.0/0.0/16_019/0000766).

\section{REFERENCES}

[1] D. Fuerst, M. Hollensteiner, A. Schrempf. A novel augmented reality simulator for minimally invasive spine surgery. vol. 46, pp. 200-204. 2014. http: //dl.acm.org/citation. cfm?id=2685617.2685645

[2] M. Hollensteiner, D. Furst, P. Augat, et al. Procedurespecific validation of artificial vertebrae. IEEE Transactions on Biomedical Engineering 65(8):18521858, 2018. DOI:10.1109/TBME.2017.2782797.

[3] S. Gomez, M. Vlad, J. Lopez, et al. Characterization and three-dimensional reconstruction of synthetic bone model foams. Materials Science and Engineering $C$ 33(6):3329-3335, 2013. DOI:10.1016/j.msec.2013.04.013

[4] H. Chen, S. Shoumura, S. Emura, Y. Bunai. Regional variations of vertebral trabecular bone microstructure with age and gender. Osteoporosis International 19(10):1473-1483, 2008. DOI:10.1007/s00198-008-0593-3

[5] T. Fila, J. Sleichrt, D. Kytyr, et al. Deformation analysis of the spongious sample in simulated physiological conditions based on in-situ compression, $4 \mathrm{~d}$ computed tomography and fast readout detector. Journal of Instrumentation 13(11), 2018. DOI:10.1088/1748-0221/13/11/C11021.

[6] M. Thompson, I. McCarthy, L. Lidgren, L. Ryd. Compressive and shear properties of commercially available polyurethane foams. Journal of Biomechanical Engineering 125(5):732-734, 2003. DOI:10.1115/1.1614820

[7] K. Calvert, K. Trumble, T. Webster, L. Kirkpatrick. Characterization of commercial rigid polyurethane foams used as bone analogs for implant testing. Journal of Materials Science: Materials in Medicine 21(5):1453-1461, 2010. DOI:10.1007/s10856-010-4024-6

[8] V. Rada, T. Fila, P. Zlamal, et al. Multi-channel control system for in-situ laboratory loading devices. In $16^{\text {th }}$ Youth Symposium On Experimental Solid Mechanics, vol. 18 of Acta Polytechnica CTU Proceedings, pp. 15-19. 2018. DOI:10.14311/APP.2018.18.0015

[9] I. Jandejsek, J. Dammer, J. Jakubek, et al. Contrast optimization in $\mathrm{x}$-ray radiography with single photon counting imagers of medipix type. Journal of

Instrumentation 7(12), 2012. DOI:10.1088/1748-0221/7/12/C12011.

[10] A. Kraemer, G. Lanza. Assessment of the measurement procedure for dimensional metrology with x-ray computed tomography. vol. 43, pp. 362-367. 2016 DOI:10.1016/j.procir.2016.02.018 